\title{
Idiopathic Pulmonary Vein Thrombosis: A Rare Presentation of Recurrent Haemontysis
}

Gayathri Prem Kumar, Pradeep Rangappa, Ipe Jacob, Rajesh Lava Kumar, Karthik Rao Intensive Care Unit, Columbia Asia Referral Hospital, Yeshwanthpur, Bangalore, Karnataka, India.

\section{Abstract:}

Idiopathic pulmonary vein thrombosis, as a cause of haemoptysis, is a rare entity published in very few medical literatures. It may mimic various other clinical conditions but has a different investigation strategy and prognosis. Pulmonary vein thrombosis is difficult to diagnose clinically, requires a high degree of suspicion and a combination of conventionally used diagnostic modalities and hence very few cases have been reported worldwide. Ours is a case of a healthy adult who presented with massive haemoptysis and was diagnosed as having idiopathic pulmonary vein thrombosis with secondary pulmonary infarction. The pulmonary veins got reanalysed spontaneously within a month of presentation and his symptoms subsided.

Key words: Pulmonary Veins, Hemoptysis, Lung Diseases, Bronchiectasis, Venous Thrombosis, Humans.

\section{Introduction}

Pulmonary vein thrombosis (PVT) is a rare but life threatening condition causing massive haemoptysis. It's rare occurrence is due to the rich network of venous collaterals in the lung [1]. It invariably occurs secondary to an inflammatory lung disease (active pulmonary Koch's or bronchiectasis) and very rarely due to other causes [2]. This entity may be under-diagnosed as the signs and symptoms are nonspecific. Even with clinical suspicion, pulmonary vein thrombosis is difficult to diagnose during life, hence its frequency may be much greater than appreciated [3]. Here we present a case that presented with haemoptysis and was diagnosed to have pulmonary vein thrombosis.

\section{Case Report}

A 33 year old gentleman, hotelier, with no known medical co-morbidities, non-smoker, social drinker, presented to us with a history of intermittent cough since 2 years and haemoptysis since 12 days. Haemoptysis increased in quantity since 4 days (about $1000 \mathrm{ml}$ - with a significant drop in haemoglobin from 16 to $8.5 \mathrm{gms} \%$ in 10 days). He had been extensively worked up at another hospital, where CT chest showed presence of infiltrates in left upper lobe with a possible cavitation and a probable embolus in a segmental branch of the left pulmonary artery. Based on the CT report, he was diagnosed with pulmonary Koch's and started on anti-tuberculosis medications empirically.

Corresponding Author: Dr. Pradeep Rangappa

Email: drpradeepr@aol.com

Received: November 27, 2014 | Accepted: January 21, 2015 | Published Online: February 15, 2015 This is an Open Access article distributed under the terms of the Creative Commons Attribution License (creativecommons.org/licenses/by/3.0) Conflict of interest: None declared | Source of funding: Nil | DOl: http://dx.doi.org/10.17659/01.2015.0018 
Clinical examination showed decreased air entry in the left infra-axillary and infra scapular areas. Laboratory investigations were normal except for haemoglobin of $9 \mathrm{gm} \%$. Chest X-ray showed haziness in the left upper and lower zone with bilateral nodular opacities. CT-PA revealed distension of left superior and inferior pulmonary veins, with low density within, which was suggestive of thrombosis [Fig.1]. There was also consolidation of the left upper and lower lobe with ground glass opacity and debris in the left main bronchus on bronchoscopy [Fig.2].

Three consecutive morning sputum samples were negative for acid fast bacillus. Thrombophilia workup (protein C, S, Anti-thrombin III, Anti phospholipid antibodies) was negative. CT abdomen, pelvis and chest were negative for malignancy. Trans-esophageal Echo was normal with no evidence of PVT in proximal pulmonary veins. The pressure gradient between the pulmonary veins and left atrium was normal.

Throughout the course of his stay in the hospital, he continued to receive anti-tubercular drugs. Though sputum culture grew Acinetobacter, he did not fulfil the criteria for hospital acquired pneumonia and hence was not started on antibiotics. Anti-coagulation was withheld on day 2 itself, as the patient had two more episodes of blood streaked sputum production. On Day 6, he coughed out a large amount of whitish, slough-like material. It was sent for histopathology, which confirmed it to be a bronchial cast with no evidence of malignant changes. Following this, his cough reduced considerably. He was discharged, at request, on antibiotics by primary physician with specific instructions to get re-admitted if the haemoptysis recurred. He was scheduled for repeat CT-PA one month later.

However, he was re-admitted the following day with another episode of massive haemoptysis

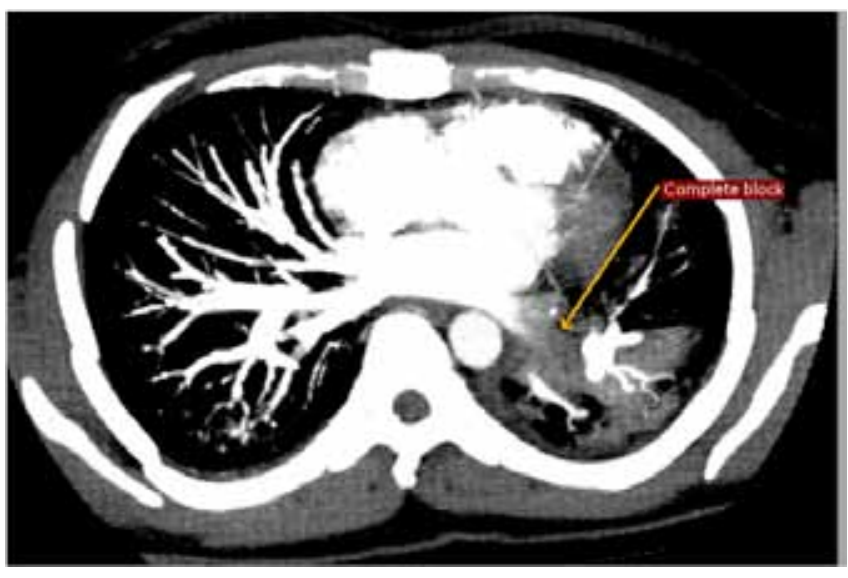

Fig.1: CT scan showing thrombosis of left inferior pulmonary vein (LIPV).

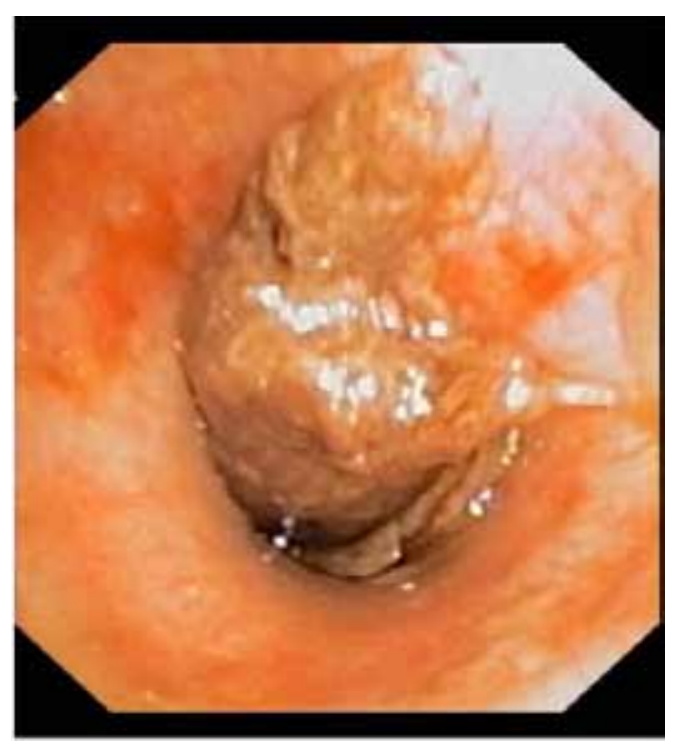

Fig.2: Debris in left main bronchus as seen on bronchoscopy.

$(\sim 500 \mathrm{ml})$. The option of pneumonectomy as a life-saving measure was offered which was not consented by the family. CT-PA was repeated and it showed complete recanalization of the pulmonary veins with significant resolution of the associated hepatisation of the left lower lobe of the lung [Fig.3]. Hence he was continued on conservative management, kept on observation for a day and 
then discharged on request. He is on regular follow up. The last follow up was in June 2013, and there have been no further episodes of haemoptysis and cough has significantly reduced.

\section{Discussion}

Idiopathic pulmonary vein thrombus (PVT) as a cause of massive haemoptysis is recorded in very few medical literature. It commonly occurs secondary to bronchogenic carcinoma, post resection of lung, lung transplantation and after radio frequency ablation for atrial fibrillation [2]. The pathophysiology of PVT is similar to mitral stenosis. PVT causes an increase in pulmonary venous pressure with compensatory pulmonary arterial vasoconstriction which causes an increase in right ventricular end diastolic pressure and right ventricular dilatation [2].

In animal studies, Wyatt and colleagues described sequential changes in the lungs after ligation of pulmonary veins (congestion, oedema, extravasation of serum and blood into alveoli leading to consolidation) [4]. Hurwitz and colleagues demonstrated complete resolution of consolidation over a period of 3-6 months, possibly due to recanalization of pulmonary veins [5].

In the largest available consecutive series, Saad et al. described severe pulmonary vein thrombosis as $70 \%$ luminal narrowing. Only $56 \%$ of these patients were symptomatic, with the most common symptom being dyspnoea followed by cough and haemoptysis. The occurrence and severity of symptoms was related to the degree of luminal narrowing and number of pulmonary veins involved [6].

Detection of pulmonary vein thrombosis is difficult because the associated symptoms like cough, dyspnoea, haemoptysis [2,7] and pleuritic chest pain are non-specific. PVT can present in two ways: acutely as pulmonary infarction with cough,

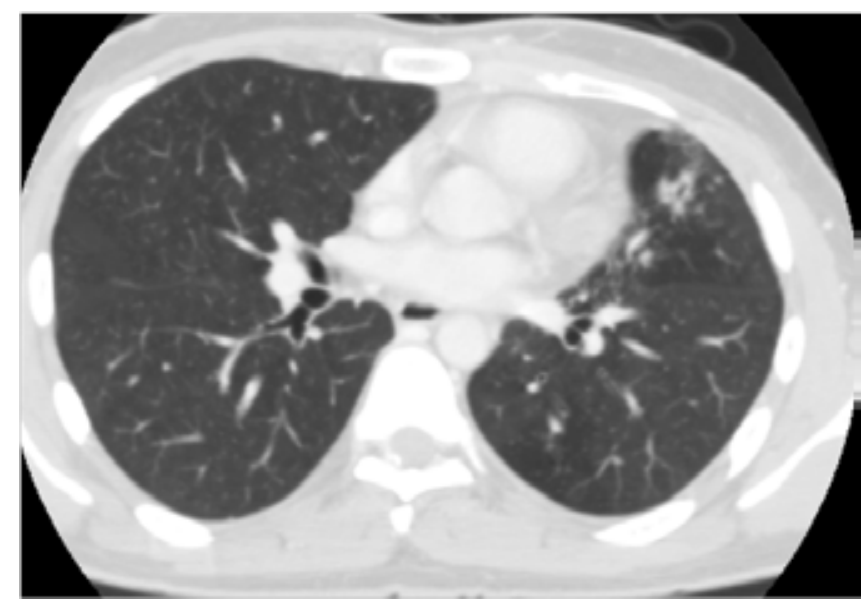

Fig.3: CT scan showing complete resolution and recanalization of thrombi in left inferior pulmonary vein (LIPV).

dyspnoea and pleuritic chest pain or in an insidious manner as progressive or recurrent pulmonary edema and pulmonary fibrosis [1]. Chest X-ray may show non-specific air space densities or opacities, which is again inconclusive. Trans-esophageal Echo can show thrombi in the large distal pulmonary veins and left atria. MR imaging can distinguish a bland thrombus from a tumour thrombus. $C T$ is a reliable, reproducible and preferred tool which can show pulmonary venous distension which is suggestive of a thrombus or can show filling defect when intravenous contrast is administered [8].

Treatment options include thrombectomy and anticoagulation in the absence of bleeding. Lobectomy or pneumonectomy are indicated when clinical deterioration occurs or when lung gangrene supervenes [5]. Life threatening complications such as pulmonary gangrene and peripheral embolization can occur when pulmonary venous occlusion is overlooked [5].

We conclude that the patient included in our study had haemoptysis due to pulmonary vein thrombosis, which was picked up on $\mathrm{CT}$ pulmonary angiogram. Haemoptysis can occur secondary to 
pulmonary tuberculosis as well, but since he had documented PVT, his haemoptysis is being attributed to PVT. The aetiology of PVT in this case is unknown as all workup for potential causes of PVT was negative. The cause for spontaneous recanalization is also uncertain in this case as the cause could not be ascertained. This case report reiterates the fact that idiopathic pulmonary vein thrombosis could be a cause to be considered in a patient presenting with massive haemoptysis.

\section{References}

1. Cavaco RA, Kaul S, Chapman T, Casaretti R, Philips B, Rhodes A, et al. Idiopathic Pulmonary Fibrosis Associated with pulmonary vein thrombosis: A case report. Cases Journal. 2009;2:9156.

2. Alexander GR, Reddi A, Reddy D. Idiopathic Pulmonary vein thrombosis: $A$ rare cause of massive hemoptysis. Ann Thorac Surg. 2009;88:281-283.
3. Kim NH, Roldan CA, Shively BK. Pulmonary vein thrombosis. Chest. 1993; 104:624-626.

4. Wyatt PJ, Burke RD, Hanlon RC. Morphology study of canine lungs after ligation of the pulmonary veins. Am J Pathol. 1953;29:291303.

5. Hurwitz A, Calabresi M, Cooke WR, Liebow AA. An experimental study of the venous collateral circulation of the lung: functional observations. J Thorac Surg. 1954;28:241-246.

6. Saad EB, Rossillo A, Saad CP, Martin DO, Bhargava $M$, Erciyes $D$. Pulmonary vein stenosis after catheter ablation of atrial fibrillation: emergence of a new clinical syndrome. Ann Intern Med. 2003;138(8):634-639.

7. Yataco J, Stoller JK. Pulmonary vein thrombosis complicating pulmonary venous thrombosis following Radiofrequency ablation. Respir Care. 2004; 49(1 2):1525-1527.

8. Selvidge SDD, Gavant ML. Idiopathic Pulmonary vein thrombosis: Detection by $C T$ and $M R$ imaging. AJR 1999;172:1639-1641. 